\title{
Antiviral drugs prioritization for COVID-19 management based on rational selection
}

\author{
Rakesh S. Joshi ${ }^{1,2, *}$, Ashok P. Giri ${ }^{1,2}$, Mahesh J. Kulkarni ${ }^{1,2}$, Mahesh Gupta ${ }^{3}$, \\ Savita Verma ${ }^{3}$, Dhruva Chaudhry ${ }^{3}$, Narendra Deshmukh ${ }^{4}$ and Anita Chugh ${ }^{4}{ }^{4}$ \\ ${ }^{1}$ Biochemical Sciences Division, CSIR-National Chemical Laboratory, Dr. Homi Bhabha Road, Pune 411 008, India \\ ${ }^{2}$ Academy of Scientific and Innovative Research (AcSIR), Ghaziabad 201 002, India \\ ${ }^{3}$ Pandit Bhagwat Dayal Sharma Post Graduate Institute of Medical Sciences, Rohtak 124 001, India \\ ${ }^{4}$ INTOX Private Limited, Pune 412 115, India
}

The SARS-CoV-2 infection has resulted in COVID-19 pandemic worldwide. It has infected around 0.1 billion individuals and caused 2 million fatalities across the globe till mid-January 2021. Drug repurposing has been utilized as the most preferred therapeutic intervention for COVID-19 mitigation due to its necessity and feasibility. To prioritize therapeutic regime against COVID-19, we used 61 antiviral drugs and their combinations. Selected molecules were subjected to virtual screening against: (i) human angiotensin-converting enzyme 2 receptor binding domain (hACE-2) which serves as an anchor for virus attachment and entry, (ii) SARS-CoV-2 RNA dependent RNA polymerase (RdRp) responsible for viral RNA replication, and (iii) SARS-CoV-2 main protease $\left(M^{\text {Pro }}\right)$ needed for viral polyprotein slab proteolytic processing. Based on docking score, pharmacodynamic and pharmacokinetic parameters, combinations of Daclatasvir, Elbasvir, Indinavir, Ledipasvir, Paritaprevir and Rilpivirine were analysed further. Our analysis suggested Sofosbuvir in combination with Ledipasvir and Daclatasvir as potential therapeutic agents for SARS-CoV-2. The combined score suggests that these combinations have superior antiSARS-CoV-2 potential than Remdesivir and other investigational drugs. The present work provides a rationale-based approach to select drugs with possible anti-SARS-CoV-2 activity for further clinical evaluation.

Keywords: Drug repurposing, hACE-2, main protease, RNA dependent RNA polymerase, SARS-CoV-2.

GLOBAL spread and infection of Severe Acute Respiratory Syndrome Coronavirus 2 (SARS-CoV-2) resulted in COVID-19 pandemic. More than 0.1 billion have been infected with a mortality rate up to $5 \%$ till the end of January 2021 (ref. 1). Due to the unavailability of a specific therapeutic regime, COVID-19 management is challenging. Drug repurposing offers a speedy solution for COVID19 mitigation. Ongoing application of repurposed drugs

*For correspondence. (e-mail: rs.joshi@ncl.res.in;

anita.chugh@intoxlab.com) aims to control symptoms of the disease or attain antiviral effect (viral replication cycle). Danoprevir, Darunavir, Favipiravir, Lopinavir/Ritonavir, Oseltamavir, Remdesivir and Umifenovir have been tested clinically against SARS-CoV-2 (ref. 2).

Furthermore, non-antiviral drugs like Camostat or Nafamostat, Chloroquine, Hydroxychloroquine, and Ivermectin have shown anti-SARS-CoV-2 potential ${ }^{3}$. Literature suggests that targeting multiple closely interacting pathogenesis-related proteins can provide effective intervention. Hence, several treatments and clinical trials used combinations of antiviral drugs for viral infection management. We selected three targets from the interaction network of pathogenesis. These targets are the virus entry point, Human angiotensin-converting enzyme 2 receptor binding domain (hACE-2), SARS-CoV-2 RNA dependent RNA polymerase ( $\mathrm{RdRp}$ ) for viral RNA replication and SARS-CoV-2 main protease $\left(\mathrm{M}^{\mathrm{Pro}}\right)$ for virus maturation $^{4}$.

SARS-CoV-2 attaches and infects human cells through hACE-2 receptor ${ }^{5,6}$. The extracellular domain of hACE-2 serves as SARS-CoV-2 spike (S) receptor. Cleavage product of $\mathrm{S}$ protein, $\mathrm{S} 1$, interacts with hACE-2 and anchors to the viral membrane by $\mathrm{S} 2$ protein $^{6-8}$. SARSCoV-2 shows strong binding ( $\sim 10$ fold) with hACE-2 compared to other coronaviruses, hence, serving as a vital target for intervention ${ }^{5}$. Multiprotein complex facilitates the replication of SARS-CoV-2 RNA genome and serves as a therapeutic target. RNA-dependent RNA polymerase (RdRp or NSP12), a cleavage product of viral polyproteins (ORF1a and ORF1b), catalyses the replication and transcription cycle of the virus. Due to its essentiality, RdRp has been explored as one of the primary targets for nucleoside analogues antivirals, e.g. Remdesivir ${ }^{9,10}$. Self-maturation and processing of viral replicase enzymes can be targeted by inhibition of papain-like main protease $\left(\mathrm{M}^{\mathrm{Pro}}\right)^{11,12}$. Due to low similarity with human proteases, inhibitors of $\mathrm{M}^{\text {Pro }}$ show minimal cross-reactivity ${ }^{11,13}$.

Molecules inhibiting viral entry, replication and maturation can have the potential anti-SARS-CoV-2 activity. Sixty one approved antiviral drugs were screened in silico for binding against selected targets. A combined activity 
score was calculated based on differential binding energy to targets. Further, pharmacokinetic and pharmacodynamic parameters were calculated to prioritize drug candidates for repurposing for COVID-19 management.

\section{Methods}

\section{Library and target molecules preparation}

Approved antivirals library was generated using threedimensional structures from PubChem ${ }^{14}$. They were checked for stereochemical properties, followed by conversion to *.pdbqt format by Autodock Tools ${ }^{15}$. The library was used for further docking studies. The necessary methodology was as described by an earlier study ${ }^{4}$.

Experimental structures of hACE-2 complexed with viral spike protein (PDB ID: 6VW1) and $\mathrm{M}^{\text {Pro }}$ (PDB ID: $6 \mathrm{Y} 2 \mathrm{~F}$ ) were downloaded from $\mathrm{PDB}^{7,11}$. After the receptor preparation process on $\mathrm{M}^{\mathrm{Pro}}$, the grid for docking simulation was set using AutoGrid program around active site residues $\mathrm{H} 41$ and $\mathrm{C} 145$ with $36 \times 56 \times 40 \AA$ dimension $^{15}$ and structure converted to *.pdbqt format. Similarly, the structure of SARS-CoV-2 RdRp (PDB: 7BTF) was prepared by generating a grid of $34 \times 34 \times 36 \AA$ dimension around RNA binding pocket ${ }^{9}$. Furthermore, the grid for hACE2 was $20 \times 38 \times 24 \AA$ spanning the viral spike protein recognition residues (K31, E35, D38, M82, K353) ${ }^{7}$. These target molecules were then further used for virtual screening.

\section{Virtual screening using combined score analysis}

The prepared receptor molecules from custom-made libraries were set for virtual screening by AutoDock Vina using default parameters ${ }^{16}$. Top hits of ligands were selected based on their docking scores. Comparative analysis of binding score and the combined score was performed using heatmap analysis, followed with hierarchical clustering and rank product analysis. Relative weightage was assigned as follows: $\mathrm{M}^{\mathrm{Pro}}=20 \%$; $\mathrm{RdRp}=$ $20 \%$ and $\mathrm{hACE}-2=60 \%$. The combined activity score was calculated as ((binding energy $\left.\mathrm{M}^{\text {Pro }} * 0.2\right)+($ binding energy RdRp *0.2) + (binding energy hACE- $2 * 0.6)$ ). Ligands with high solubility and bioavailability were further taken for interaction analysis. A detailed methodology of interaction analysis was as described by an earlier study ${ }^{4}$.

\section{Results and discussion}

Outcomes of in silico screening of antiviral drugs demonstrate several molecules' potential to intervene SARSCoV-2 infection cycle. They can potentially offer avenues to manage COVID-19. Remdesivir, Lopinavir/
Ritonavir (Kaletra) ${ }^{17,18}$, Favipiravir ${ }^{19}$ and Umifenovir are among top antivirals being focused on for COVID-19 management. Nucleotide analogue, Remdesivir, serve as broad-spectrum antiviral against RNA viruses like Coronaviridae. Initial preclinical and clinical studies indicated Remdesivir causing a reduction in viral load through RdRp inhibition ${ }^{10}$. Remdesivir exhibited binding energy $-7.8,-8.2$ and $-7.2 \mathrm{kcal} / \mathrm{mol}$ against $\mathrm{hACE}-2, \mathrm{M}^{\text {Pro }}$ and RdRp respectively (Figure 1). Remdesivir has a higher

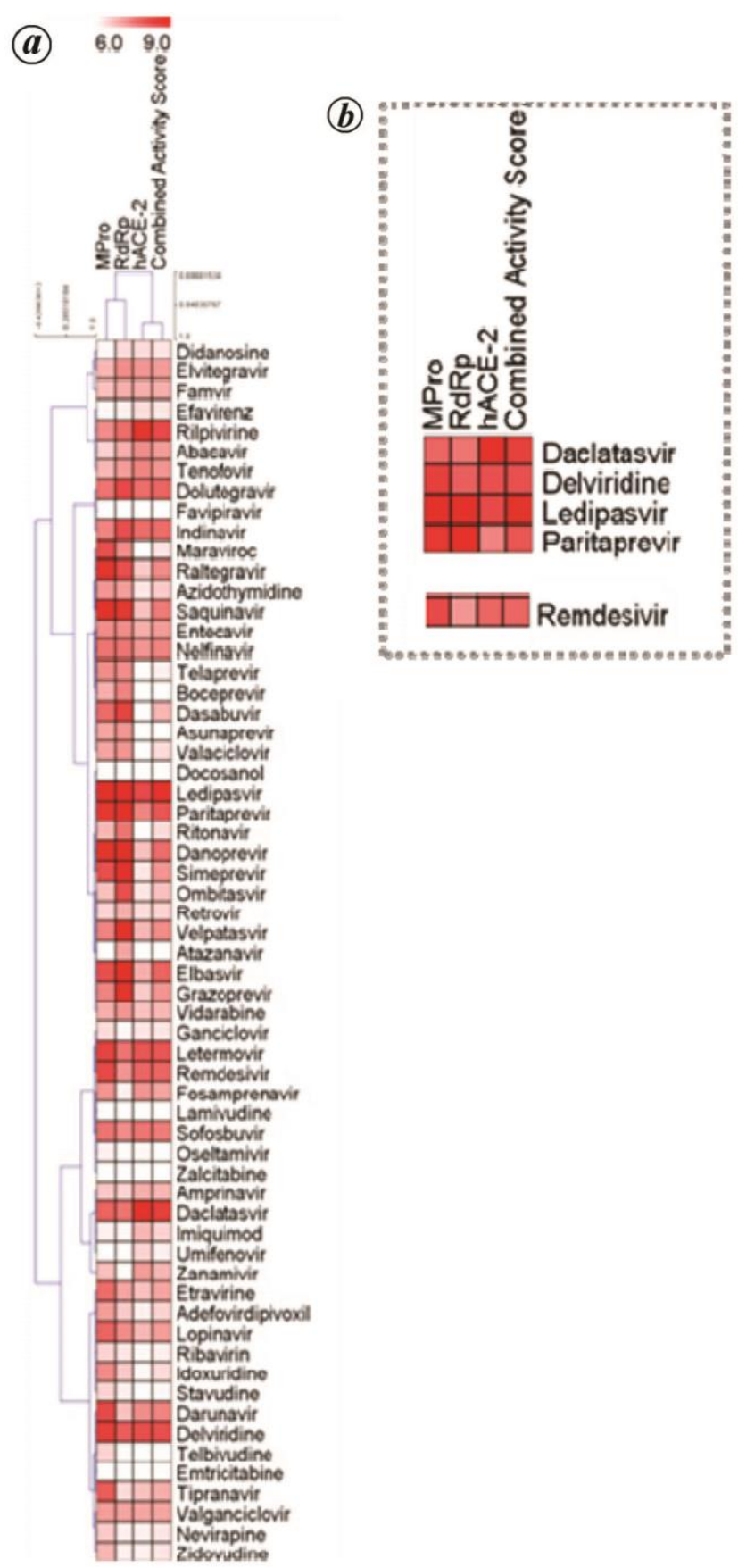

Figure 1. $\boldsymbol{a}$, Heatmap with hierarchial clustering for binding and combined score analysis of screened antivirals. $\boldsymbol{b}$, Ranking of binding score indicating top molecules. 
Table 1. Drug candidates exhibiting strong binding to $\mathrm{M}^{\mathrm{Pro}} / \mathrm{RdRp} / \mathrm{hACE}-2$

\begin{tabular}{|c|c|c|c|c|}
\hline Target & $\begin{array}{l}\text { Binding energy range* } \\
(\mathrm{kcal} / \mathrm{mol})\end{array}$ & Drug & $\begin{array}{l}\text { Binding energy } \\
(\mathrm{kcal} / \mathrm{mol})\end{array}$ & $\begin{array}{l}\text { Combined activity } \\
\text { score }\end{array}$ \\
\hline \multirow[t]{5}{*}{$\overline{\mathrm{M}^{\mathrm{Pro}}}$} & -3.2 to -10.2 & Remdesivir & -8.2 & 7.8 \\
\hline & & Ledipasvir & -9.4 & 8.8 \\
\hline & & Saquinavir & -9.0 & 7.5 \\
\hline & & Danoprevir & -8.7 & 7.7 \\
\hline & & Raltegravir & -8.7 & 7.3 \\
\hline \multirow[t]{5}{*}{$\mathrm{RdRp}$} & -3.2 to -9.4 & Remdesivir & -7.2 & 7.8 \\
\hline & & Ledipasvir & -10.2 & 8.8 \\
\hline & & Elbasvir & -10.0 & 7.8 \\
\hline & & Danoprevir & -9.8 & 7.7 \\
\hline & & Paritaprevir & -9.4 & 8.0 \\
\hline \multirow[t]{4}{*}{ hACE-2 } & -2.8 to -8.9 & Remdesivir & -7.8 & 7.8 \\
\hline & & Daclatasvir & -8.9 & 8.4 \\
\hline & & Rilpivirine & -8.7 & 8.2 \\
\hline & & Ledipasvir & -8.2 & 8.8 \\
\hline
\end{tabular}

Drugs exhibiting low binding energy to either $\mathrm{M}^{\mathrm{Pro}} / \mathrm{RdRp} / \mathrm{hACE}-2$ are shortlisted. *Range of 61 antiviral drugs screened.

Table 2. Binding energy and combined activity scores for approved antiviral drugs exhibiting hACE-2 binding better than or comparable to Remdesivir

\begin{tabular}{lcccc}
\hline & \multicolumn{3}{c}{ Binding energy $(\mathrm{kcal} / \mathrm{mol})$} & Combined activity \\
\cline { 2 - 4 } Drug & $\mathrm{M}^{\text {Pro }}$ & RdRp & hACE-2 & score \\
\hline Combined activity & score significantly & better than Remdesivir \\
Ledipasvir & -9.4 & -10.2 & -8.2 & 8.8 \\
Daclatasvir & -7.8 & -7.6 & -8.9 & 8.4 \\
& & & & \\
Combined activity & score comparable to Remdesivir & \\
Rilpivirine & -7.3 & -7.7 & -8.7 & 8.2 \\
Delviridine & -8.4 & -7.9 & -8.1 & 8.1 \\
Paritaprevir & -8.6 & -9.4 & -7.4 & 8.0 \\
Letermovir & -8.3 & -7.6 & -8.0 & 8.0 \\
Dolutegravir & -7.6 & -8.2 & -7.7 & 7.8 \\
Remdesivir* & -8.2 & -7.2 & -7.8 & $7.8 *$ \\
Indinavir & -7.4 & -8.1 & -7.7 & 7.7 \\
Sofosbuvir* & -7.6 & -7.3 & -7.6 & 7.5 \\
Darunavir & -8.2 & -6.7 & -7.3 & 7.4 \\
Abacavir* & -6.5 & -6.9 & -7.3 & $7.4 *$ \\
Tenofovir* & -6.8 & -7.1 & -7.4 & $7.1 *$ \\
\hline These drugs & & &
\end{tabular}

*These drugs are converted intracellularly to active metabolites. The binding energy of known key active metabolite present intracellularly was considered for $\mathrm{M}^{\text {Pro }}$ and $\mathrm{RdRp}$ for combined activity score determination.

combined activity score compared to selected repurposed antiviral and non-antiviral drugs against SARS-CoV-2. Selected drugs and their metabolites binding energy and combined activity are provided in Supplementary Table 1.

Binding energy and combined activity score for selected antiviral drugs are shown in Supplementary Table 2. The binding energy of approved antiviral drugs for respective target is outlined in Table 1.

Ledipasvir, Elbasvir, Danoprevir, Saquinavir and Paritaprevir showed strong binding with $(<-9 \mathrm{kcal} / \mathrm{mol})$ either target. Candidates having lower binding energy against hACE-2 than Remdesivir were shortlisted for further evaluation (Table 2).

Daclatasvir and Ledipasvir depicted better combined activity score than Remdesivir (>8.2) and strong hACE-2 binding $(\leq-8.2 \mathrm{kcal} / \mathrm{mol})$. Daclatasvir and Ledipasvir are used as combination therapy against Hepatitis $\mathrm{C}$ Virus $(\mathrm{HCV})$ infection ${ }^{20-22}$. They inhibit HCV RNA replication and assembly of virions by blocking non-structural Protein 5A (NS5A). They are FDA approved against $\mathrm{HCV}$ infections as the fixed-dose combination (FDC) with Sofosbuvir ${ }^{22,23}$

From in silico analysis, Ledipasvir was found to be a top hit for drug repurposing. For $\mathrm{M}^{\mathrm{Pro}}$ and RdRp, it has a binding energy of $<-9 \mathrm{kcal} / \mathrm{mol}$ and against hACE-2 $\simeq 8.2 \mathrm{kcal} / \mathrm{mol}$. Amongst the screened antivirals, Daclatasvir binds strongly with hACE-2 and interfere with its binding to spike protein of SARS-CoV-2. Daclatasvir interaction with the binding pocket of targets is shown in Figure 2, and pharmacokinetic overview of these drugs is summarized in Table 3.

Remdesivir (GS-5734) is used as a reference molecule in the current study. It is prodrug form of adenosine analogue (GS-441524) and can be intracellularly metabolized to an active nucleoside triphosphate $(\mathrm{NTP})^{10}$. Pro- and metabolite forms of Remdesivir exhibit strong binding against RdRp and $\mathrm{M}^{\text {Pro }}$ (Supplementary Table 1). The prodrug can bind to hACE-2 weakly for a prolonged time due to its extended half-life, while, the parent molecule, Remdesivir, has effective hACE-2 inhibition for a shorter duration due to short half-life (Table 3).

The half-life for Ledipasvir and Daclatasvir is $47 \mathrm{~h}$ and 12 to $15 \mathrm{~h}$ respectively. Due to longer half-life, they can have a long-term binding with hACE-2. Additionally, due to significant intracellular concentrations, they show noteworthy binding against $\mathrm{RdRp}$ and $\mathrm{M}^{\text {Pro }}$. Hence, 
RESEARCH ARTICLES

Table 3. Pharmacokinetic properties of lead candidates and reference drug (Remdesivir*)

\begin{tabular}{|c|c|c|c|}
\hline & Remdesivir* & Ledipasvir & Daclatasvir \\
\hline Status & Investigational & Approved & Approved \\
\hline Indication (*in clinics) & SARS-COV-2 & $\begin{array}{l}\text { Chronic HCV genotype } 1 \mathrm{a}, 1 \mathrm{~b}, 4,5 \\
\text { and } 6 \text { infection in combination } \\
\text { with Sofosbuvir (Harvoni) }\end{array}$ & $\begin{array}{l}\text { Chronic HCV genotype } 1,3 \text { and } 4 \\
\text { infection in combination with } \\
\text { Sofosbuvir, Ribavirin or interferon }\end{array}$ \\
\hline $\begin{array}{l}\text { The key known target for } \\
\text { the approved indication }\end{array}$ & $\begin{array}{l}\text { RdRp inhibition by } \\
\text { triphosphate metabolite } \\
\text { (NTP) }\end{array}$ & $\begin{array}{l}\text { Prevent hyperphosphorylation of } \\
\text { NS5A }\end{array}$ & $\begin{array}{l}\text { Prevent hyperphosphorylation of } \\
\text { NS5A }\end{array}$ \\
\hline Bio-availability & Not available & $76 \%$ & $67 \%$ \\
\hline Protein binding & Not available & $>99.8 \%$ & $99 \%$ \\
\hline Elimination half-life & $\begin{array}{l}0.4 \mathrm{~h} \text { parent (non-human } \\
\text { primate (NHP)) } 20 \mathrm{~h} \text { for } \\
\text { NTP metabolite in } \\
\text { humans, } 14 \mathrm{~h} \text { in NHP }\end{array}$ & $47 \mathrm{~h}$ (median terminal) & $12-15 \mathrm{~h}$ \\
\hline
\end{tabular}

Daclatasvir and Ledipasvir are considered for repurposing against COVID-19.

Route of administration for Remdesivir is intravenous, whereas Daclatasvir and Ledipasvir, are orally administered. Combinations of Ledipasvir and Daclatasvir with Sofosbuvir are clinically approved. In docking analysis, Sofosbuvir also exhibited high combined activity score of 7.5. Thus, the additive putative synergistic effect could be expected in these approved antiviral drug combinations and they provide merit over other drugs' combinations for COVID-19 management. Due to moderate to better protein binding capacity of these molecules, high drug concentration becomes available to bind against the extracellular hACE-2 target. Furthermore, the circulating half-life of Sofosbuvir is $0.4 \mathrm{~h}$. It is metabolized as triphosphate form GS-46103 (2'-deoxy-2'- $\alpha$-fluoro- $\beta$-Cmethyluridine- $5^{\prime}$-triphosphate) and dephosphorylated metabolite GS 331007 subsequently, which has an elimination half-life of $27 \mathrm{~h}$. Thus, Sofosbuvir can bind for a longer time to intracellular targets such as RdRp and $\mathbf{M}^{\text {Pro }}$ (Supplementary Table 3).

In the case of approved Daclatasvir and Sofosbuvir combination with Ribavarin for HCV treatment, Ribavarin did not exhibit good combined activity score (6.2) against SARS-CoV-2 targets. Hence, Ribavarin was not considered for prioritization (Supplementary Table $2)^{23}$. Similarly, another fixed-dose combination (FDC) of Daclatasvir with Asunaprevir against HCV showed low combined activity score (5.7) for Asunaprevir. Hence Asunaprevir was dropped from further analysis.

Combined scores for Rilpivirine, Viridine, Paritaprevir, Letermovir and Dolutegravir are better than Remdesivir (7.8 to 8.3$)^{24}$. Rilpivirine binds strongly to hACE-2 and Paritaprevir showed higher binding to $\mathrm{RdRp}$ and $\mathrm{M}^{\text {Pro }}$. All these drugs can bind to hACE-2 in pro-form and have high protein binding (Supplementary Table 4). Nonnucleoside reverse transcriptase inhibitors (NNRTI) like
Rilpivirine and Delviridine are approved against HIV-1 infections ${ }^{25-27}$. As a second-line therapy drug Delaviridine is inconvenient due to its dosing schedule, it is therefore dropped from further evaluation.

Paritaprevir in combination with Ombitasvir, Dasabuvir, Ritonavir, and Ribavirin is used to treat $\mathrm{HCV}^{28}$. Most of these antivirals exhibited low binding score against all targets and thus have negligible anti-SARSCoV-2 potential.

Abacavir, Darunavir, Indinavir, Sofosbuvir and Tenofovir showed high binding to SARS-CoV-2 target. Short half-life, moderate protein binding (60\%), acceptable safety profile and strong hACE- 2 binding $(-7.7 \mathrm{kcal} / \mathrm{mol})$ makes Indinavir a potential agent. Indinavir and Ritonavir FDC is used for HIV treatment. Ritonavir has a low combined activity score, but it blocks the intracellular conversion of Indinavir and thus prolongs its half-life. Hence, the FDC of Indinavir and Ritonavir can be antiSARS-CoV-2 (ref. 29). The development of new FDC of these drugs for intravenous use can be considered in critically ill patients (Supplementary Table 1).

Short half-life $(1.54 \pm 0.63 \mathrm{~h})$ and moderate protein binding $(50 \%)$, with a binding energy of $-7.3 \mathrm{kcal} / \mathrm{mol}$ against hACE-2 suggest the potential of Abacavir for effective intervention. Abacavir and its metabolite Carbovir triphosphate depicted high binding energy against $\mathbf{M}^{\text {Pro }}$ and RdRp (Supplementary Table 3$)^{30}$. In HIV infection, Abacavir combination with Lamivudine, Zidovudine and Dolutegravir is used for the treatment. The Lamivudine and Zidovudine have combined activity scores 5.3 and 6.3 respectively. Due to good combined activity score, high protein binding and half-life of $14 \mathrm{~h}$, Dolutegravir and Abacavir combination are preferred over a combination with Lamivudine or Zidovudine for COVID-19. Elbasvir has the potential against SARS-CoV-2 replication and maturation due to high binding to $\mathrm{RdRp}$ $(-10 \mathrm{kcal} / \mathrm{mol})$ and combined activity score of 7.8 . This 


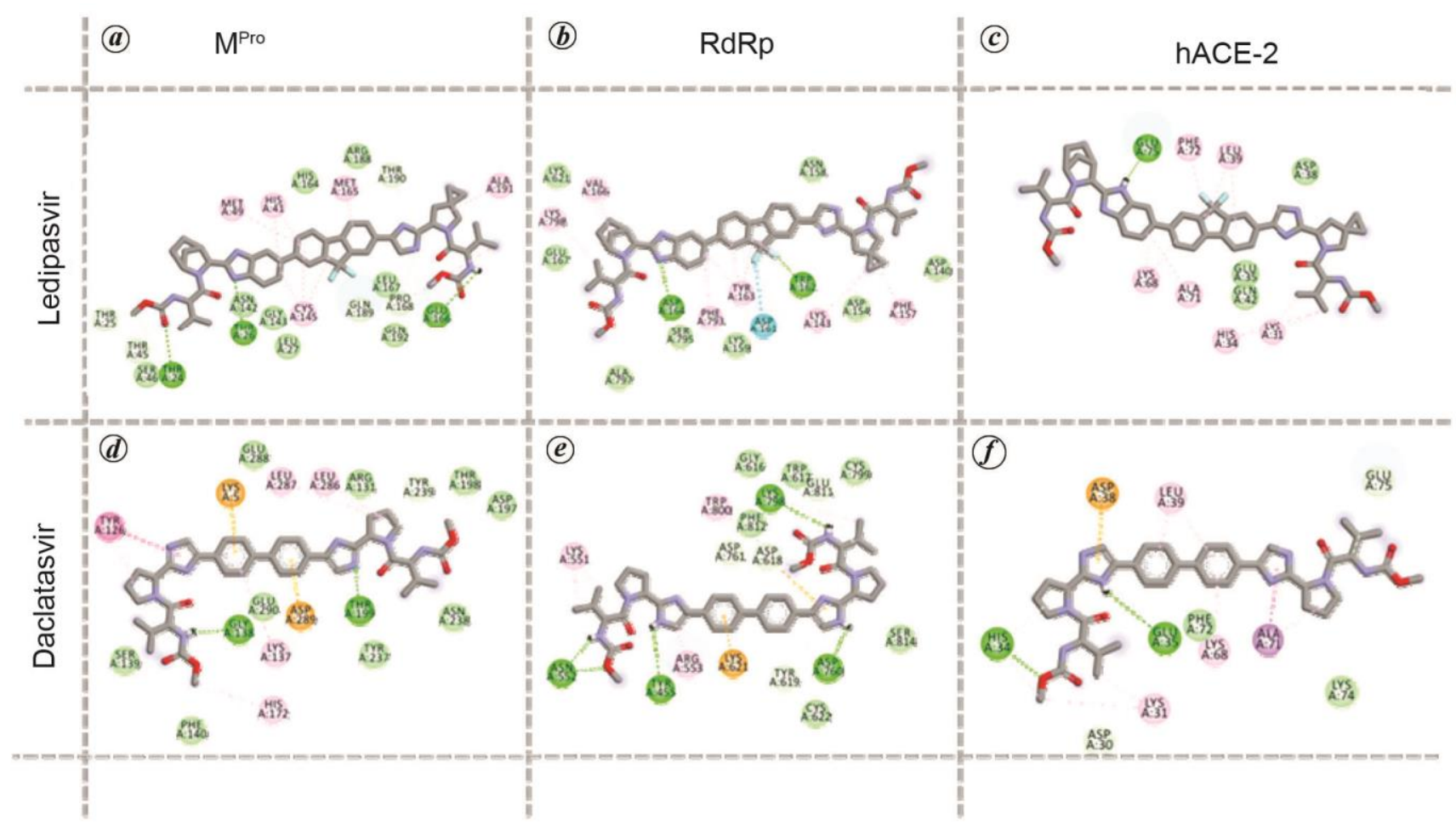

Figure 2. Molecular interaction of Ledipasvir with binding pocket of $(\boldsymbol{a}) \mathrm{M}^{\mathrm{Pro}}$, (b) RdRp and (c) hACE-2. Likewise, Daclatasvir also showed stronger binding and multiple interactions with binding sites of $(\boldsymbol{d})$ MPro, $(\boldsymbol{e}) \operatorname{RdRp}$ and $(\boldsymbol{f})$ hACE-2.

drug has a half-life (geometric mean) of $24 \mathrm{~h}$ and can be evaluated as a candidate drug for COVID-19. Elbasvir is a direct-acting antiviral which inhibits HCV NS5A protein and approved as the FDC with Grazoprevir or Ribavarin and is a part of combination therapy to treat $\mathrm{HCV}^{22,31}$. Grazoprevir exhibits a combined activity score of 7.2 and shows low binding energy for $\mathrm{RdRp}$ $(-8.7 \mathrm{kcal} / \mathrm{mol})$, superior to Ribavarin. Hence, FDC of Elbasvir with Grazoprevir can be checked for COVID-19 management.

Danoprevir, NS3/4A protease inhibitor approved for $\mathrm{HCV}$, exhibits distinctly better binding for $\mathrm{M}^{\text {Pro }}$ $(-8.7 \mathrm{kcal} / \mathrm{mol})$ and $\operatorname{RdRp}(-9.8 \mathrm{kcal} / \mathrm{mol})$. Danoprevir half-life and protein binding information are not available in the public domain; also, it showed poor binding to hACE-2. It does not offer an advantage over other lead candidates. Another protease inhibitor, Saquinavir, exhibits high binding to $\mathrm{M}^{\text {Pro }}(-9.0 \mathrm{kcal} / \mathrm{mol})$. Due to its low combined activity score (7.5) and poor bioavailability, it is not considered for prioritization. Earlier in silico studies have identified two non-antiviral drugs Ergotamine and Ubrogepant as potential anti-SARS-CoV-2 agents (Supplementary Table 5) $)^{32,33}$. These molecules exhibited the potential to bind to all three targets. Binding energy at the crucial target of interest hACE-2 for Ubrogepant was $-7.0 \mathrm{kcal} / \mathrm{mol}$. Ergotamine showed strong binding against all three targets as compared to Ledipasvir (Supplementary Table 5), while Daclatasvir showed stronger hACE-2 binding compared to Ergotamine ${ }^{32}$. It has been observed that cardiovascular drugs like ACE inhibitors and angiotensin receptor antagonists do not show affinity toward hACE-2 (ref. 34). Hence, this further strengthens the case for Daclatasvir and Ledipasvir's prioritization as lead candidates for COVID-19 over Ergotamine and other similar drugs.

Cleavage of $\mathrm{S} 2$ protein is catalysed by a human cell surface serine protease, TMPRSS $2^{6,35}$. Therefore, along with hACE-2, TMPRSS2 can be considered for effective targeting of viral replication and maturation. Camostat and Nafamostat have demonstrated inhibition of TMPRSS2 $2^{5,36}$. Hence, Camostat and Nafamostat and their combinations with antiviral drugs with high binding for hACE-2 such as Daclatasvir and Rilpivirine can offer synergistic effects against SARS-CoV-2.

We suggest that molecules with potential for strong hACE-2 binding with RdRp and $\mathrm{M}^{\text {Pro }}$ interaction can be repurposed against SARS-CoV-2. Additionally, due to better protein binding and long half-life, suggested drug/ drug combinations can exhibit activity better than other drugs under investigation. Further, shortlisted drugs are orally administered and thus offer an advantage over others. There is a need for systematic preclinical and clinical assessment for these drugs and their FDC for anti-SARSCoV-2 activity. These repurposed drugs might provide potential antiviral effect against SARS-CoV-2 better than other drugs under trials and tested investigational drugs. 


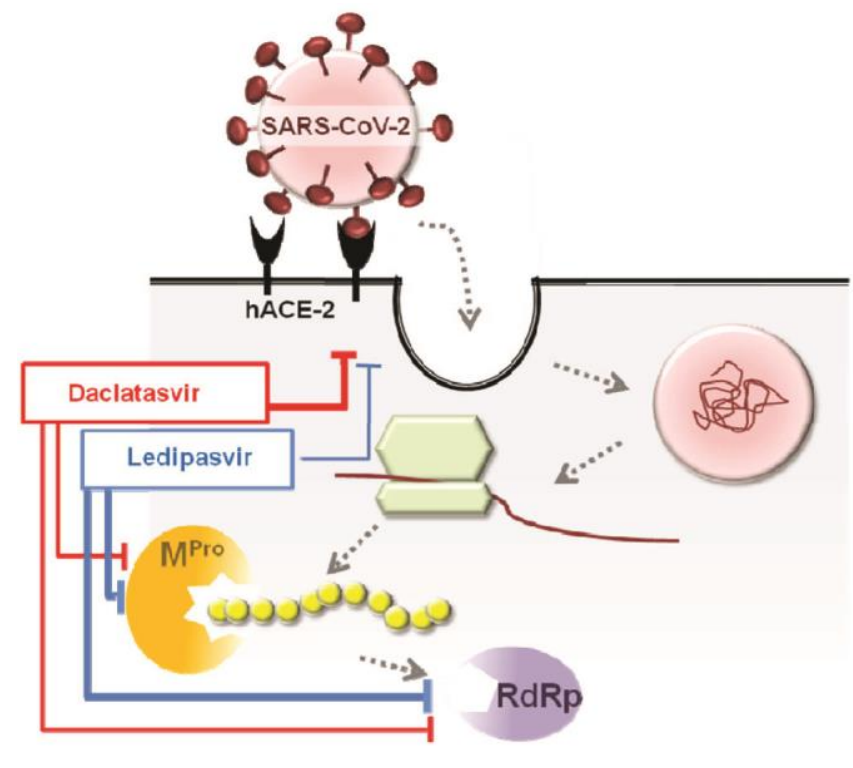

Figure 3. Schematic representation of predicted actions of Ledipasvir and Daclatasvir against multiple targets in SARS-CoV-2. Ledipasvir prominently targeting $\mathrm{M}^{\mathrm{Pro}}$ and $\mathrm{RdRp}$ is indicated by the thick blue lines and binding moderately to hACE-2 is illustrated by relatively thin blue line. In the case of Daclatasvir, binding is strong with hACE-2 and is indicated by a thick red line. Daclatasvir binds moderately to $\mathrm{M}^{\text {Pro }}$ and $\mathrm{RdRp}$ which is indicated by thin red lines.

\section{Conclusion}

We virtually identified drugs with potential to bind to multiple targets like SARS-CoV-2 $\mathrm{M}^{\text {Pro }}$ and RdRp; and hACE-2 (Figure 3). These repurposed drugs are likely to have anti-SARS-CoV-2 activity by impacting virus entry, replication and maturation.

Daclatasvir, Elbasvir, Indinavir, Ledipasvir, Paritaprevir and Rilpivirine were predicted as potential anti-SARSCoV-2 based on combined activity score, pharmacokinetic and pharmacodynamic parameters. Ledipasvir and Daclatasvir emerged as lead candidates with high combined activity scores and prolonged half-life, ensuring significant extracellular hACE-2 engagement along with RdRp and $\mathrm{M}^{\mathrm{Pro}}$. With good safety profile and oral administration of Ledipasvir, Daclatasvir and other drugs selected through this screening, can provide an advantage over others. These drugs and their FDCs can be considered for systematic fast track preclinical and clinical evaluation for COVID-19 management. Our findings provide a scientific rationale for applying Ledipasvir and Daclatasvir in combination with Sofosbuvir for COVID19 management. Recent initial clinical trials data from Iran with Ledipasvir and Daclatasvir in combination with Sofosbuvir against COVID-19 are encouraging. Based on our analysis and available preclinical and clinical data, we recommend prioritization and aggressive perusal of clinical evaluation of these drug combinations.
1. WHO, Coronavirus disease. World Health Organization, 2020, 2633.

2. Thorlund, K., Dron, L., Park, J., Hsu, G., Forrest, J. I. and Mills, E. J., A real-time dashboard of clinical trials for COVID-19. Lancet Digit. Health, 2020, 7500, 2019-2020.

3. Shaffer, L., 15 drugs being tested to treat COVID-19 and how they would work. Nat. Med., 2020

4. Joshi, R. S. et al., Discovery of potential multi-target-directed ligands by targeting host-specific SARS-CoV-2 structurally conserved main protease. J. Biomol. Struct. Dyn., 2020, 5, $1-16$.

5. Hoffmann, M. et al., SARS-CoV-2 cell entry depends on ACE2 and TMPRSS 2 and is blocked by a clinically proven protease inhibitor. Cell, 2020, 181, 1-10.

6. Walls, A. C., Park, Y. J., Tortorici, M. A., Wall, A., McGuire, A. T. and Veesler, D., Structure, function, and antigenicity of the SARS-CoV-2 spike glycoprotein. Cell, 2020, 181, 281-292.e6.

7. Shang, J. et al., Structural basis of receptor recognition by SARSCoV-2. Nature, 2020, 581, 1-8.

8. Li, F., Structure, function, and evolution of coronavirus spike proteins. Annu. Rev. Virol., 2016, 3, 237-261.

9. Gao, Y. et al., Structure of the RNA-dependent RNA polymerase from COVID-19 virus. Science, 2020, 7498, 1-9.

10. Amirian, E. S. and Levy, J. K., Current knowledge about the antivirals remdesivir (GS-5734) and GS-441524 as therapeutic options for coronaviruses. One Health, 2020, 9, 100128.

11. Zhang, L. et al., Crystal structure of SARS-CoV-2 main protease provides a basis for design of improved $\alpha$-ketoamide inhibitors. Science, 2020, 3405, 1-9.

12. Krichel, B., Falke, S., Hilgenfeld, R. and Redecke, L., Processing of the SARS-CoV pp1a/ab nsp7-10 region. Biochem. J., 2020, 477, 1009-1019.

13. Anand, K., Ziebuhr, J., Wadhwani, P., Mesters, J. R. and Hilgenfeld, R., Coronavirus main proteinase (3CLpro) structure: basis for design of anti-SARS drugs. Science, 2003, 300, 17631767.

14. Kim, S. et al., PubChem 2019 update: improved access to chemical data. Nucleic Acids Res., 2018, 47, D1102-D1109.

15. Morris, G. and Huey, R., AutoDock4 and AutoDockTools4: automated docking with selective receptor flexibility. J. Comput. Chem., 2009, 30, 2785-2791.

16. Trott, O. and Olson, A. J., AutoDock Vina: improving the speed and accuracy of docking with a new scoring function, efficient optimization, and multithreading. J. Comput. Chem., 2010, 31, 455-461.

17. Cao, B. et al., A trial of lopinavir-ritonavir in adults hospitalized with severe COVID-19. N. Engl. J. Med., 2020, 1-13.

18. Muralidharan, N., Sakthivel, R., Velmurugan, D. and Gromiha, M. M., Computational studies of drug repurposing and synergism of lopinavir, oseltamivir and ritonavir binding with SARS-CoV-2 protease against COVID-19. J. Biomol. Struct. Dyn., 2020, 1-7 (at press)

19. Chen, C. et al., Favipiravir versus arbidol for COVID-19: a randomized clinical trial. medRxiv, 2020, 2020.03.17.20037432.

20. Kumari, R. and Nguyen, M. H., Fixed-dose combination of sofosbuvir and ledipasvir for the treatment of chronic hepatitis $\mathrm{C}$ genotype 1. Exp. Opin. Pharmacother., 2015, 16, 739-748.

21. Belema, M. et al., Hepatitis C virus NS5A replication complex inhibitors: the discovery of daclatasvir. J. Med. Chem., 2014, 57, 2013-2032.

22. Chung, R., AASLD/IDSA HCV guidance: recommendations for testing, managing, and treating hepatitis C. Clin. Liver Dis., 2018, 12, 117.

23. Younossi, Z. M. et al., Patient-reported outcomes in chronic hepatitis $\mathrm{C}$ patients with cirrhosis treated with Sofobuvircontaining regimens. Hepatology, 2015, 61, 1798-1808. 
24. Xia, Q., Radzio, J., Anderson, K. S. and Sluis-Cremer, N., Probing non-nucleoside inhibitor-induced active-site distortion in HIV-1 reverse transcriptase by transient kinetic analyses. Protein Sci., 2007, 16, 1728-1737.

25. Fernández-Montero, J. V., Vispo, E., Anta, L., de Mendoza, C. and Soriano, V., Rilpivirine: a next-generation non-nucleoside analogue for the treatment of HIV infection. Exp. Opin. Pharmacother., 2012, 13, 1007-1014.

26. Fantauzzi, A. and Mezzaroma, I., Dolutegravir: clinical efficacy and role in HIV therapy. Ther. Adv. Chronic Dis., 2014, 5, 164177.

27. Zamora, F. and Ogbuagu, O., Dolutegravir/rilpivirine for the treatment of HIV-1 infection. HIV AIDS (Auckl), 2018, 10, 215224 .

28. Klibanov, O. M., Gale, S. E. and Santevecchi, B., Ombitasvir/ paritaprevir/ritonavir and dasabuvir tablets for hepatitis $\mathrm{C}$ virus genotype 1 infection. Ann. Pharmacother., 2015, 49, 566-581.

29. Hull, M. W. and Montaner, J. S. G., Ritonavir-boosted protease inhibitors in HIV therapy. Ann. Med., 2011, 43, 375-388.

30. Faletto, M. B., Miller, W. H., Garvey, E. P., St. Clair, M. H., Daluge, S. M. and Good, S. S., Unique intracellular activation of the potent anti-human immunodeficiency virus agent 1592 U89. Antimicrob. Agents Chemother., 1997, 41, 1099-1107.

31. Bell, A. M., Wagner, J. L., Barber, K. E. and Stover, K. R., Elbasvir/ grazoprevir: a review of the latest agent in the fight against hepatitis C. Int. J. Hepatol., 2016, 2016.
32. Vishal, M., Pravin, D., Himani, G., Nilam, V., Urvisha, B. and Rajesh, P., Drug repurposing of approved drugs elbasvir, ledipasvir, paritaprevir, velpatasvir, antrafenine and ergotamine for combating COVID-19. chemRxiv, 2020.

33. Omotuyi, O. et al., The disruption of SARS-CoV-2 RBD/ACE-2 complex by ubrogepant is mediated by interface hydration. Preprints, 2020, 2020030466.

34. Cure, E. and Cumhur Cure, M., Angiotensin-converting enzyme inhibitors and angiotensin receptor blockers may be harmful in patients with diabetes during COVID-19 pandemic. Diabetes Metab. Syndr., 2020, 14, 349-350.

35. Hasan, A. et al., A review on the cleavage priming of the spike protein on coronavirus by angiotensin-converting enzyme- 2 and furin. J. Biomol. Struct. Dyn., 2020, 1-13 (at press).

36. Yamamoto, M. et al., The anticoagulant nafamostat potently inhibits SARS-CoV-2 infection in vitro: an existing drug with multiple possible therapeutic effects. Viruses, 2020, 12, 629; doi:10.3390/v12060629.

ACKNOWLEDGEMENT. This work is supported by BIRAC grant (BT/COVID0079/02/20).

Received 1 July 2020; revised accepted 18 November 2020

doi: $10.18520 / \mathrm{cs} / \mathrm{v} 120 / \mathrm{i} 9 / 1464-1470$ 